\title{
Espiritualidade entre os profissionais da saúde que atuam na linha de frente do enfrentamento à COVID-19, em um município do Sudoeste da Bahia e sua correlação com a qualidade de vida, transtornos mentais e medo da COVID
}

Spirituality among health professionals who work on the front line of confronting COVID-19, in a municipality in the Southwest of Bahia, and its correlation with quality of life, mental disorders and fear of COVID

Espiritualidad entre los profesionales de la salud que trabajan en primera línea del enfrentamiento al COVID-19, en un municipio del Suroeste de Bahía, y su correlación con la calidad de vida, los trastornos mentales y el miedo al COVID

Viviane Moreira dos Santos Teixeira ORCID: https://orcid.org/0000-0002-0869-7178 Universidade Estadual de Montes Claros, Brasil E-mail: vimoreirat34@gmail.com

Heloísa Ribeiro Alves

ORCID: https://orcid.org/0000-0003-1412-0487 Faculdade Independente do Nordeste, Brasil E-mail: heloisaralves@ outlook.com Laiane Ferreira da Silva ORCID: https://orcid.org/0000-0002-0069-1337 Universidade Estadual de Montes Claros, Brasil E-mail: laianesilva489@gmail.com Antônio Alvimar Souza

ORCID: https://orcid.org/0000-0002-6046-7580 Universidade Estadual de Montes Claros, Brasil E-mail: aalvimars@hotmail.com

Walter de Freitas Filho

ORCID: https://orcid.org/0000-0001-9502-0968 Universidade Estadual de Montes Claros, Brasil E-mail: walterdefreitas00@gmail.com

Andréa Maria Eleutério de Barros Lima Martins ORCID: https://orcid.org/0000-0001-7865-1733 Universidade Estadual de Montes Claros, Brasil E-mail: martins.andreamebl@gmail.com

\section{Resumo}

A COVID-19 disseminou-se por todo o mundo por um crescimento exponencial no número de casos, tendo sido declarada pela OMS como uma pandemia em março de 2020. Dentre os diferentes mecanismos de enfrentamento aos desafios impostos pela pandemia, os profissionais de saúde têm a espiritualidade e a religiosidade como suporte no enfrentamento das situações de incertezas e adversidade, sendo o binômio uma fonte de conforto e de entendimento. Foi verificado a incidência da espiritualidade entre os profissionais da saúde que atuam na linha de frente do enfrentamento à COVID-19 em um município do sudoeste da Bahia e sua correlação com a qualidade de vida, o medo da COVID e o desenvolvimento de transtornos mentais leves. Trata-se de um estudo transversal, de caráter descritivo e de abordagem quantitativa, realizado entre 152 profissionais. As condições sócio-econômico-demográficas foram avaliadas por um questionário elaborado previamente pelos pesquisadores, a variável espiritualidade, religiosidade e crenças pessoais avaliada por meio da aplicação do Instrumento WHOQOL-SRBP, as variáveis transtornos mentais comuns, qualidade de vida e medo da COVID foram investigadas, respectivamente, por meio do QSG, SF-12 e Medo da COVID. Os dados foram submetidos à análise descritiva e bivariada, por meio do coeficiente de correlação de postos de Spearman. Responderam aos questionários do estudo 147 profissionais (96,71\%), destacando-se os escores apresentados pelo WHOQOL-SRBP, entre 48 e 127, IC [99,07;105,01] e pelo QSG, 9 e 36, IC [20,72;24,35]. Concluiu-se que a espiritualidade está relacionada ao menor índice de transtornos mentais comuns e à maior qualidade de vida. 
Palavras-chave: Infecções por coronavírus; Pessoal de saúde; Saúde ocupacional; Espiritualidade; Qualidade de vida.

\begin{abstract}
COVID-19 has spread throughout the world due to an exponential growth in the number of cases, having been declared by the WHO as a pandemic in March 2020. Among the different mechanisms to face the challenges posed by the pandemic, health professionals have spirituality and religiosity as support in coping with situations of uncertainty and adversity, the binomial being a source of comfort and understanding. The incidence of spirituality among health professionals who work on the front line of confronting COVID-19 was verified in a municipality in the southwest of Bahia and its correlation with quality of life, fear of COVID and the development of mild mental disorders. This is a cross-sectional, descriptive study with a quantitative approach, carried out among 152 professionals. Socio-economicdemographic conditions were assessed using a questionnaire previously prepared by the researchers, the variable spirituality, religiosity and personal beliefs assessed through the application of the WHOQOL-SRBP instrument, the variables common mental disorders, quality of life and fear of COVID were investigated, respectively, through the QSG, SF-12 and Fear of COVID. Data were submitted to descriptive and bivariate analysis using Spearman's rank correlation coefficient. 147 professionals answered the study questionnaires (96.71\%), highlighting the scores presented by the WHOQOL-SRBP, between 48 and 127, CI [99.07;105.01] and by the QSG, 9 and 36, CI [20.72;24.35]. It was concluded that spirituality is related to a lower rate of common mental disorders and a higher quality of life.
\end{abstract}

Keywords: Coronavirus infections; Health personnel; Occupational health; Spirituality; Quality of life.

\title{
Resumen
}

El COVID-19 se ha extendido por todo el mundo debido a un crecimiento exponencial en el número de casos, habiendo sido declarado por la OMS como pandemia en marzo de 2020. Entre los diferentes mecanismos para enfrentar los desafíos que plantea la pandemia, los profesionales de la salud cuentan con espiritualidad y la religiosidad como soporte para afrontar situaciones de incertidumbre y adversidad, siendo el binomio fuente de consuelo y comprensión. Se verificó la incidencia de la espiritualidad entre los profesionales de la salud que trabajan en la primera línea del enfrentamiento al COVID-19 en un municipio del suroeste de Bahía y su correlación con la calidad de vida, el miedo al COVID y el desarrollo de trastornos mentales leves. Se trata de un estudio descriptivo transversal con abordaje cuantitativo, realizado entre 152 profesionales. Se evaluaron las condiciones socioeconómicas-demográficas mediante un cuestionario previamente elaborado por los investigadores, se evaluó la variable espiritualidad, religiosidad y creencias personales mediante la aplicación del instrumento WHOQOL-SRBP, se evaluaron las variables trastornos mentales comunes, calidad de vida y miedo al COVID, investigados, respectivamente, a través del QSG, SF-12 y Fear of COVID. Los datos se sometieron a análisis descriptivo y bivariado utilizando el coeficiente de correlación de rangos de Spearman. 147 profesionales respondieron los cuestionarios del estudio (96,71\%), destacando las puntuaciones presentadas por el WHOQOL-SRBP, entre 48 y 127, IC [99,07; 105,01] y por el QSG, 9 y 36, IC [20,72; 24,35]. Se concluyó que la espiritualidad está relacionada con una menor tasa de trastornos mentales comunes y una mayor calidad de vida.

Palabras clave: Infecciones por coronavirus; Personal de salud; Salud ocupacional; Espiritualidad; Calidad de vida.

\section{Introdução}

O primeiro coronavírus a causar infecção em seres humanos foi registrado na década de 60 (Kendall; Bynoe; Tyrrell, 1962). A partir de então, foram descobertas outras cepas distintas, as quais podem provocar desde infecções respiratórias leves até os casos mais graves, como pneumonia e envolvimento renal, podendo ainda afetar o trato gastrointestinal, fígado, rins e cérebro (Su et al., 2016). Em dezembro de 2019 foi identificado na cidade de Wuhan, na China, a presença do vírus Severe Acute Respiratory Syndrome Coronavirus 2 (SARS-CoV-2), como agente causador de casos de pneumonia grave, (Li; DE Clercq, 2020), trata-se de um novo coronavírus humano (Yee et. al., 2020), o qual foi nomeado provisoriamente pela Organização Mundial de Saúde (OMS) como o novo coronavírus de 2019, ou 2019-nCoV (Chan et al., 2020; Lu et al., 2020; Wu et al., 2020).

Sendo causada pelo SARS-CoV-2, a doença do coronavírus, Corona Vírus Disease-19 (COVID-19) (Zhang, 2020; Ferrer, 2020), apresenta-se como uma doença infecciosa que vem se alastrando por todo o mundo por um crescimento exponencial no número de casos, tendo sido declarada como uma pandemia em março de 2020 pela OMS (Ferrer, 2020), apresentando uma rápida disseminação e número elevado de mortes, haja vista o risco de o vírus causar dificuldade respiratória 
aguda grave (Zhang et al., 2020). Não se restringindo somente ao sistema respiratório, a COVID-19 afeta também o sistema hematológico, onde o vírus acomete os glóbulos vermelhos, causando um quadro de hipóxia gradativa (Sant'ana, Silva, Vasconcelos, 2020). No decurso da doença, destaca-se ainda a produção elevada de células do sistema imunológico, responsáveis pela resposta inflamatória, podendo afetar os tecidos pulmonares, renais e cardíacos (Cespedes; Souza, 2020). Ademais, o SARS-CoV-2 tem o potencial de se replicar em células do sistema nervoso, podendo ocasionar em manifestações neurológicas (Chu et. al., 2020), bem como complicações cardíacas (Strabelli; Uip, 2020) e vasculares, dentre elas os eventos trombóticos (Casella, 2020).

Dentre os diferentes mecanismos de enfrentamento aos desafios impostos pela pandemia da COVID-19, considerando-se a busca pelo bem-estar, os profissionais de saúde têm a espiritualidade como uma importante aliada no exercício do cuidado em todas as suas extensões, sendo esta uma dimensão particular do ser humano, que atua como medida de suporte tanto na relação com o sofrimento oriundo do processo da doença, quanto na necessidade de fortalecimento diante dos sentimentos adversos como o medo, angústia, insegurança e frustração (Tavares, 2020), da mesma forma, a religiosidade também tem sido utilizada frente à COVID-19 como suporte no enfrentamento das situações de incertezas e adversidade, sendo uma fonte de conforto e de entendimento (Bentzen, 2020).

Deste modo, as questões relacionadas à espiritualidade e religiosidade sempre estiveram ligadas com a saúde, havendo importante influência destas no dia a dia dos profissionais da saúde, através de mecanismos como a fé, a qual atua positivamente gerando esperança (Gerone, 2016). Assim, a influência do binômio espiritualidade/religiosidade frente aos aspectos relacionados à saúde tem sido atualmente tema de interesse nas discussões entre diferentes autores, os quais avaliam a saúde através do conceito de integralidade e identificam a atuação positiva, tanto da espiritualidade quanto da religiosidade, sobre a condição de bem-estar (Alves et al., 2010).

No que se refere ao conceito de espiritualidade e religiosidade, vale ressaltar que as duas estão atreladas, mas não trazem em si o mesmo significado. Para tanto, a religiosidade está relacionada a crenças específicas à determinada doutrina, comum a um determinado grupo de pessoas, havendo a prática de cultos ou rituais atrelados a fé e podendo estar atrelada à crendice, enquanto a espiritualidade consiste em um sentimento originado do relacionamento com o transcendente, nas diferentes dimensões da existência de cada indivíduo, manifestando-se de forma individualizada, podendo estar associada a crenças religiosas (Underwood, 1999). Outrossim, ambas consistem em características intrínsecas à condição subjetiva do homem, havendo integração na busca pela resposta aos seus anseios e na determinação do sentido da vida, bem como na relação sobre a saúde de cada pessoa (Melo et al., 2015).

Diante do atual cenário pandêmico vivenciado, a saúde ocupacional dos profissionais de saúde deve ser repensada e discutida, a fim de se propor mecanismos de enfrentamento, além da preservação da saúde, visto a exposição aos diversos tipos de riscos no ambiente de trabalho em que esta classe se encontra e a importância fundamental destes no combate à doença (Fiho et al., 2020).

Destarte, tendo em vista as dificuldades enfrentadas pelos profissionais que atuam nos serviços de saúde, no acolhimento e cuidados aos pacientes portadores da COVID- 19, tanto nas unidades hospitalares quanto na atenção primária à saúde, faz-se necessário a realização de novos estudos em contextos específicos, a fim de contribuir para a compreender a importância da espiritualidade no contexto do exercício das atividades de trabalho destes profissionais. Neste sentido, o objetivo deste estudo foi verificar a incidência da espiritualidade entre os profissionais da saúde que atuam na linha de frente do enfrentamento à COVID - 19 em um município do sudoeste da Bahia e sua correlação com a qualidade de vida, o medo da COVID e o desenvolvimento de transtornos mentais leves. 


\section{Metodologia}

Trata-se de um estudo descritivo, transversal, sendo o fator e o efeito analisados em um mesmo período de tempo (Rouquayrol, 1994) e de abordagem quantitativa, propondo-se a analisar dados e indicadores passíveis de serem observados por meio de variáveis (MINAYO; SANCHES, 1993), realizado entre os trabalhadores da saúde atuantes na linha de frente do atendimento aos pacientes com casos suspeitos ou confirmados da COVID-19 em um município do sudoeste da Bahia, compreendendo os técnicos de enfermagem e os profissionais de nível superior das unidades hospitalares e de atenção primária à saúde. Produto de uma dissertação que avalia o tema espiritualidade, religiosidade e crenças pessoais entre profissionais da saúde em tempos de COVID-19.

Para a realização da pesquisa foram selecionadas quatro instituições hospitalares e todas as unidades de atenção primária à saúde (APS) do município, compreendendo 23 unidades na zona urbana e 19 unidades na zona rural. A lista com os nomes e telefones dos profissionais foi disponibilizada pelo setor de recursos humanos de cada instituição hospitalar e pela Comissão Municipal de Ensino e Pesquisa da Secretaria Municipal de Saúde do Município, em se tratando da APS, sendo incluídos os profissionais de ambos os sexos, com idade entre 18 e 59 anos, regularmente contratados ou concursados que foram designados para atuar no manejo ambulatorial, clínico ou intensivo aos pacientes suspeitos e/ou confirmados para COVID-19. Foram excluídos aqueles que tenham sido transferidos para setor que não seja destinado ao atendimento de paciente com COVID-19, bem como os que se encontravam em atestado médico, afastamento temporário de suas atividades laborais ou férias.

No total, foram elencados 713 profissionais $(\mathrm{N}=428$ nas instituições hospitalares e $\mathrm{N}=285$ na APS), a partir dos quais procedeu-se um planejamento amostral por meio da seleção de uma amostra aleatória simples (AAS), tendo em vista o cálculo amostral para população finita, considerando a estimativa para proporções da ocorrência dos eventos ou doenças em $50 \%$ da população $(0,50)$, erro amostral tolerável de até 7,5\% $(0,075)$ e nível de confiança de $95 \%(Z=1,96)$, sendo estimado uma amostra de 138 foi acrescentado uma taxa de não resposta de 10\%, totalizando 152 profissionais. A partir desta amostra, foi conduzido um sorteio de uma amostra aleatória simples no banco de dados organizado previamente no software Statistical Package Social Science (SPSS®), versão 25.0.

Os dados foram coletados durante o período de novembro 2020 à março 2021, por meio de um questionário autoaplicável, composto por questões elaboradas pelos pesquisadores para a avaliação das características sociodemográficas, e por instrumento validados no Brasil WHOQOL-SRBP, SF-12, QSG e Medo da COVID, a fim de se obter o objetivo proposto. Os questionários foram enviados aos profissionais, pelos próprios pesquisadores, via WhatsApp, após contato prévio e aceitação pelos profissionais.

As variáveis independentes estudadas foram a condições sócio-econômico-demográficas e a espiritualidade.

As condições sócio-econômico-demográficas foram avaliadas por um questionário elaborado previamente pelos pesquisadores, compreendendo 18 questões, as quais compreedem: nome completo, localidade, data de nascimento, idade em anos, profissão, sexo biológico, sexualidade e gênero, escolaridade, estado civil, raça, ocupação atual, renda familiar mensal bruta, alteração na renda familiar durante o período de isolamento, números de pessoas que moram com o entrevistado, tipo de domicílio em que reside, número de cômodos e número de varandas e áreas externas.

A variável Espiritualidade, religiosidade e crenças pessoais, foi estudada mediante a aplicação do Instrumento de Qualidade de Vida da Organização Mundial da Saúde (OMS), World Health Organization Quality of Life (WHOQOL), por meio de um módulo desenvolvido pela OMS, especificamente para a avaliação das questões voltadas à Espiritualidade, Religiosidade e Crenças Pessoais, Spirituality, Religiouness e Personal Beliefs (domínio SRPB) (Fleck; Skevington,2007). 
Trata-se de um instrumento já validado internacionalmente (Catré et. al., 2014) e traduzido para o português brasileiro, sendo composto por oito fatores: conexão a ser ou força espiritual, sentido na vida, admiração, totalidade e integração, força espiritual, paz interior, esperança e otimismo, e fé; cada fator contém quatro itens correspondentes, totalizando 32 itens. (Panzini et. al., 2011). Os itens foram enumerados a partir do 0 (zero), sendo 0 "nada", 1 "muito pouco", 2 "mais ou menos", 3 "bastante" e 4 "extremamente", assim, os escores totais do WHOQOL-SRBP podem variar de 0 a 128, sendo que quanto maior o escore maior a presença da espiritualidade.

Foram consideradas como variáveis dependentes a presença de transtornos mentais comuns, a qualidade de vida e o medo da COVID, as quais foram avaliadas por meio da aplicação de instrumentos já validados no Brasil, sendo, respectivamente, Questionário de Saúde geral (QSG-12), 12-Item Short-Form Health Survey (SF-12) e Escala de Medo da COVID-19 (EMC-19) (Borges; Argolo, 2002; Camelier, 2004; Barros et. al., 2021).

O QSG-12 consiste num instrumento composto por 12 questões, apresentando quatro opções de respostas em cada uma, numa escala de concordância, tendo sido desenvolvido com a finalidade de detectar a presença de transtornos mentais comuns ou não psicóticos, sendo um instrumento comumente utilizado para medir o bem-estar psicológico (Borges; Argolo, 2002). As questões variaram entre 0 “ absolutamente não", 1 “ muito menos que de costume", 2 " mais que de costume" e 3 "muito mais que de costume", podendo os escores totais variar de 0 a 36, a fim de se obter a pior situação em escores mais baixos, os valores das variáveis QSG2, QSG5, QSG6, QSG9, QSG10 e QSG 11 foram invertidos de 0 para 3, 1 para 2,2 para 1 e 3 para 0, desse modo, quanto maior o escore do QSG menor a presença de transtornos mentais comuns.

O SF-12 trata-se de um instrumento utilizado para avaliar as distintas dimensões da qualidade de vida, sendo constituído por 12 questões, compostas por um grupo de respostas organizadas em escala tipo Likert, as quais avaliam a função física, aspecto físico, dor, saúde geral, vitalidade, função social, aspecto emocional e saúde mental, considerando os aspectos equivalentes à saúde dos entrevistados nas últimas quatro semanas (Silveira et. al. 2013) As respostas foram organizadas em 0 "excelente", 1 " muito boa", 2 "boa", 3 " regular" e 4 " ruim", para caracterizar a saúde; 0 "sim, dificulta muito", 1 " sim, dificulta um pouco" e 2 "não, não dificulta", sobre dificuldades decorrentes da saúde; 0 "sim" e 1 "não", no que diz respeito aos problemas com o trabalho ou atividades do dia-a-dia; 0 "não, nem um pouco", 1 "um pouco", 2 "moderadamente", 3 " bastante" e 4 " extremamente" sobre problemas com dor durante o trabalho e 0 "todo o tempo", 1 " a maior parte do tempo", 2 "uma boa parte do tempo", 3 " alguma parte do tempo", 4 " uma pequena parte do tempo" e 5 "nem um pouco de tempo", para verificar sentimentos de calma, serenidade e energia nas últimas quatro semanas. Os escores do SF-12 podem variar de 0 a 32 . A fim de se obter a pior situação de qualidade de vida em escores mais baixos, os valores das variáveis QV1 foram invertidos de 0 para 3, 1 para 2, 2 para 1 e 3 para 0 ; em QV8 houve a inversão dos valores 0 para 4, 1 para 3, 3 para 1 e 4 para 0 (o valor 2 foi mantido); nas variáveis QV9 e QV10 houve a inversão de 0 para 5, 1 para 4, 2 para 3, 3 para 2, 4 para 1 e 5 para 0. Assim, quanto maior o escore melhor a qualidade vida.

O EMC-19 apresenta-se como um questionário composto por 12 questões e sete itens em sua versão original, utilizado na literatura para o estudo do medo da COVID-19 no período perinatal (Barros et. al., 2021), o mesmo foi utilizado nesta pesquisa para embasar a mensuração da variável medo da COVID, sendo um elaborado um questionário onde foram adicionadas questões relacionadas à saúde ocupacional. As respostas consistem em 0 " discordo totalmente", 1 "discordo", 2 "nem concordo, nem discordo", 3 "concordo", 4 "concordo totalmente", podendo apresentar escores que variem de 0 a 36, neste caso, quanto maior o escore maior o medo da COVID.

Para a análise de dados empregou-se a versão 25 do software Statistical Package Social Science (SPSS®). Todas as variáveis citadas foram trabalhadas de forma categórica e tratadas por uma abordagem quantitativa, procedendo-se as 
análises descritivas e bivariadas, após cálculo das variáveis pelo método aditivo simples, respeitando os princípios da bioestatística. As variáveis que tiveram seus valores invertidos foram recodificadas em variáveis diferentes antes de serem calculadas.

Esta pesquisa faz parte do projeto "Saúde Ocupacional entre trabalhadores da saúde em tempos de COVID”, aprovado pelo Comitê de Ética em Pesquisa da Universidade Estadual de Montes Claros - CEP/Unimontes, sob Parecer n ${ }^{\circ}$ 4.101.281. Todos os participantes assinaram o termo de consentimento livre e esclarecido e todas as instituições envolvidas assinaram o termo de concordância para participação na pesquisa.

\section{Resultados e Discussão}

Dos 152 profissionais selecionados, $147(96,71 \%)$ responderam aos questionários e 5 (3,28\%) recusaram-se a participar. Dentre os 147 respondentes, foi feito uma seleção dos casos no software SPSS, sendo descartados os que não responderam às questões válidas do instrumento usado para a verificação da espiritualidade, religiosidade e crenças pessoais, o World Health Organization Quality of Life - Spirituality, Religiouness e Personal Beliefs (WHOQOL-SRPB), resultando em um banco de dados com 111 profissionais. Sendo assim, a taxa de resposta ao instrumento foi de 73,02 \% .

$\mathrm{Na}$ Tabela 1 estão descritos os dados sociodemográficos e econômicos dos profissionais de saúde participantes do estudo.

Tabela 1 - Dados Socioeconômicos, demográficos e caracterização dos profissionais de saúde participantes do estudo, Vitória da Conquista, 2020/2021.

\begin{tabular}{|c|c|c|}
\hline $\begin{array}{l}\text { Variável } \\
\text { Idade (anos) }\end{array}$ & \multicolumn{2}{|c|}{$\begin{array}{c}\text { Média } \pm \text { Desvio Padrão } \\
36,69 \pm 7,15\end{array}$} \\
\hline & $\mathbf{N}$ & $\%$ \\
\hline \multicolumn{3}{|l|}{ Faixa etária } \\
\hline $21-31$ anos & 27 & 24,3 \\
\hline $32-37$ anos & 34 & 30,6 \\
\hline $38-42$ anos & 25 & 22,5 \\
\hline $43-57$ anos & 23 & 20,7 \\
\hline \multicolumn{3}{|l|}{ Sexo } \\
\hline Feminino & 87 & 78,4 \\
\hline Masculino & 24 & 21,6 \\
\hline \multicolumn{3}{|l|}{ Raça } \\
\hline Branca & 44 & 39,6 \\
\hline Amarela & 3 & 2,7 \\
\hline Negra & 14 & 12,6 \\
\hline Parda & 47 & 42,3 \\
\hline \multicolumn{3}{|l|}{ Estado Civil } \\
\hline Casado (a) & 57 & 51,4 \\
\hline União consensual & 14 & 12,6 \\
\hline Desquitado (a), divorciado (a), separado (a), ex- união consensual & 5 & 4,5 \\
\hline Solteiro (a) & 33 & 29,7 \\
\hline \multicolumn{3}{|l|}{ Escolaridade } \\
\hline Ensino médio & 42 & 37,8 \\
\hline Ensino Superior & 68 & 61,3 \\
\hline \multicolumn{3}{|l|}{ Principal Ocupacão } \\
\hline Funcionário público & 29 & 26,1 \\
\hline Empregado de empresa privada & 71 & 64,0 \\
\hline Empresário / empregador & 1 & 9 \\
\hline Profissional liberal / autônomo & 6 & 5,4 \\
\hline \multicolumn{3}{|l|}{ Renda } \\
\hline Menor que R\$3.000,00 & 68 & 61,3 \\
\hline $\mathrm{R} \$ 3.001,00$ à $\mathrm{R} \$ 10.000,00$ & 30 & 27,0 \\
\hline Maior que $\mathrm{R} \$ 10.000,00$ & 7 & 6,3 \\
\hline
\end{tabular}




\begin{tabular}{lll}
\hline Profissão & & \\
Médico (a) & 8 & 7,2 \\
Enfermeiro (a) & 42 & 37,8 \\
Psicólogo (a) & 1 &, 9 \\
Fisioterapeuta & 9 & 8,1 \\
Nutricionista & 1 &, 9 \\
Assistente Social & 2 & 1,8 \\
Farmacêutico (a) & 2 & 1,8 \\
Técnico (a) de Enfermagem & 41 & 36,9 \\
Auxiliar de Enfermagem & 1 &, 9 \\
Odontólogo & 3 & 2,7 \\
\hline
\end{tabular}

Fonte: Dados da pesquisa (2021).

Na Tabela 2, são apresentadas as medidas descritivas das respostas de cada item do instrumento "World Health Organization Quality of Life - Spirituality, Religiousness and Personal Beliefs” (WHOQOL-SRPB), sendo possível verificar a frequência dos 32 itens que compõem os 8 domínios.

Tabela 2 - Caracterização dos profissionais de saúde do município de Vitória da Conquista quanto ao WHOQOL-SRPB nos anos de 2020/2021.

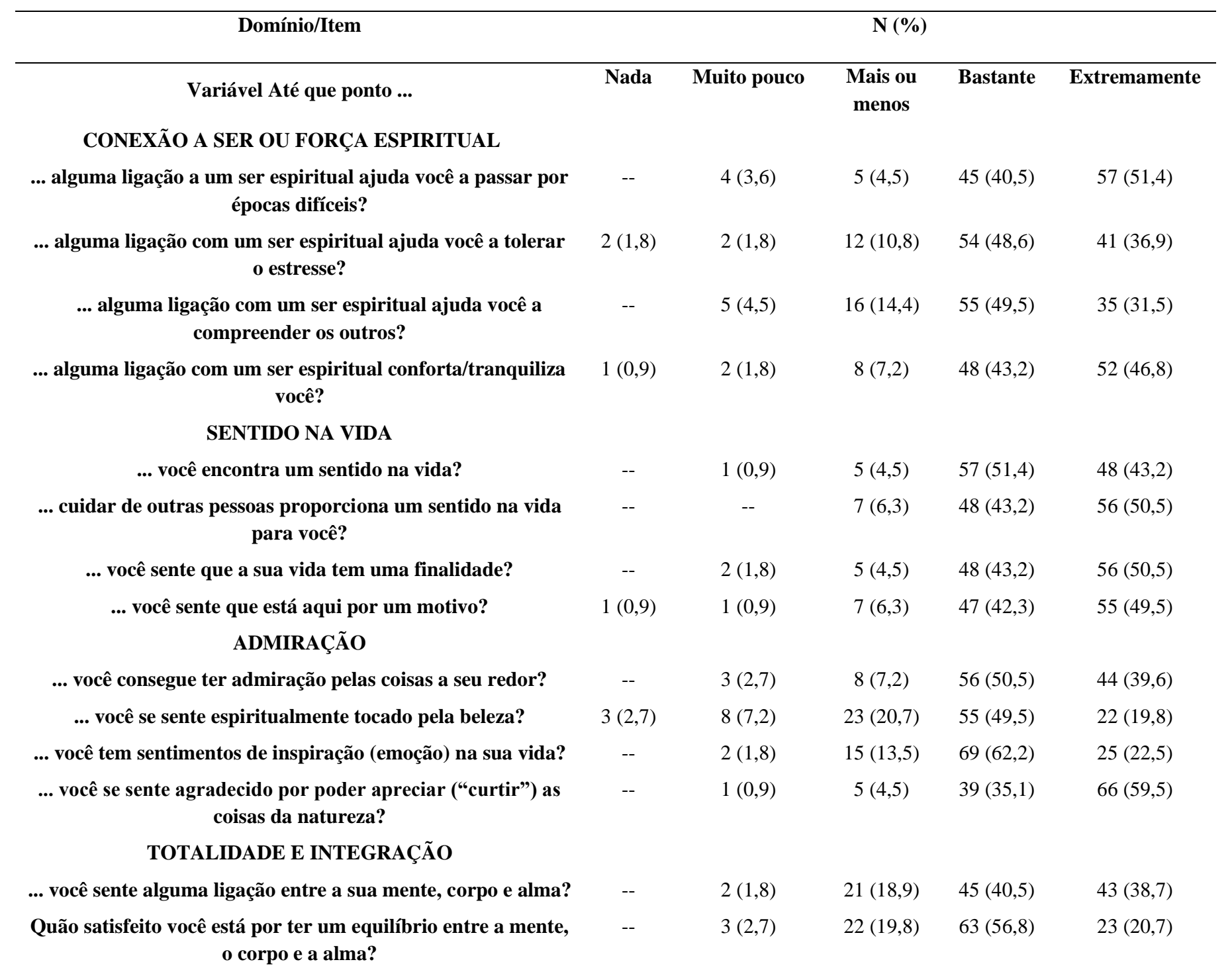


... você sente que a maneira em que vive está de acordo com o que você sente e pensa?

O quanto as suas crenças ajudam-no a criar uma coerência (harmonia) entre o que você faz, pensa e sente?

Até que ponto você sente força espiritual interior?

Até que ponto você pode encontrar força espiritual em épocas difíceis?

O quanto a força espiritual o ajuda a viver melhor?

Até que ponto a sua força espiritual o ajuda a se sentir feliz na vida?

\section{PAZ INTERIOR}

Até que ponto você se sente em paz consigo mesmo?

Até que ponto você tem paz interior?

O quanto você consegue sentir paz quando você necessita disso?

Até que ponto você sente um senso de harmonia na sua vida? ESPERANÇA E OTIMISMO

Quão esperançoso você se sente?

Até que ponto você está esperançoso com a sua vida? Até que ponto ser otimista melhora a sua qualidade de vida?

O quanto você é capaz de permanecer otimista em épocas de incerteza?

FÉ

Até que ponto a fé contribui para o seu bem-estar?

Até que ponto a fé lhe dá conforto no dia-a-dia?

Até que ponto a fé lhe dá força no dia-a-dia?

Até que ponto a fé o ajuda a gozar (aproveitar) a vida?

--

$1(0,9)$

$32(28,8) \quad 58(52,3)$

$20(18,0)$

$2(1,8)$

$11(9,9)$

$69(62,2)$

$29(26,1)$

$--\quad 2(1,8)$

$8(7,2)$

$56(50,5)$

$45(40,5)$

$1(0,9) \quad 2(1,8)$

$7(6,3)$

$52(46,8)$

$49(44,1)$

$1(0,9) \quad 2(1,8)$

$7(6,3)$

$54(48,6)$

$47(42,3)$

$1(0,9)$

$15(13,5)$

$54(48,6)$

$41(36,9)$

$\begin{array}{ccccc}-- & 1(0,9) & 20(18,0) & 64(57,7) & 26(23,4) \\ -- & 3(2,7) & 18(16,2) & 65(58,6) & 25(22,5) \\ -- & 2(1,8) & 22(19,8) & 71(64,0) & 16(14,4) \\ -- & 2(1,8) & 19(17,1) & 73(65,8) & 17(15,3) \\ & & & & \\ -- & -- & 16(14,4) & 59(53,2) & 36(32,4) \\ -- & 1(0,9) & 12(10,8) & 54(48,6) & 44(39,6) \\ -- & 1(0,9) & 12(10,8) & 52(46,8) & 46(41,4) \\ 1(0,9) & 4(3,6) & 29(26,1) & 71(64,0) & 6(5,4)\end{array}$

$\begin{array}{lllll}1(0,9) & 2(1,8) & 6(5,4) & 44(39,6) & 58(52,3) \\ 1(0,90 & 2(1,8) & 6(5,4) & 47(42,3) & 55(49,5) \\ 1(0,9) & 2(1,8) & 6(5,4) & 49(44,1) & 53(47,7) \\ 1(0,9) & 4(3,6) & 11(9,9) & 62(55,9) & 33(29,7)\end{array}$

-- Sem informação

Fonte: Dados da pesquisa (2021).

No domínio conexão a ser ou força espiritual, verifica-se a importância atribuída pelos profissionais de saúde do município de Vitoria da Conquista à ligação a um ser espiritual como mecanismo que os auxilie a passar por épocas difíceis $(51,4 \%)$, a compreender os outros $(49,5 \%)$ e a tolerar o estresse $(48,6 \%)$, assumido, portanto, um papel protetor neste cenário crítico consequente à pandemia. Neste sentido, o binômio religiosidade/espiritualidade tem sido utilizado para auxiliar na compreensão e enfrentamento das adversidades oriundas da pandemia da COVID-19, sendo os problemas de ordem emocional e as diversas mudanças no contexto social, cultural e familiar em decorrência do adoecimento e morte pela doença, gerando um sentimento de responsabilidade coletiva e auxiliando no ajustamento nos diferentes contextos e necessidades (Scorsolini-comin et. al. 2020).

Com relação aos domínios sentido na vida e admiração, a maior parte dos profissionais entrevistados afirmou, respectivamente, ter um sentido na vida $(51,4 \%)$, sentir que a sua vida tem uma finalidade $(43,2 \%)$, que cuidar de outras pessoas lhes proporciona um sentido na vida $(43,2 \%)$ e que possuem sentimentos de inspiração (emoção) na sua vida (62,2\%). Assim, a religiosidade/espiritualidade além de ser utilizada como uma ferramenta para a manutenção da saúde, poderá também auxiliar na adaptação e no planejamento de estratégias que tragam sentido às diferentes vivências oriundas do contexto 
pandêmico (Scorsolini-comin et. al. 2020). No contexto geral da assistência à saúde, especificamente nos serviços da linha de frente ao atendimento da COVID 19, faz-se necessário a utilização de mecanismos que fortaleçam os profissionais envolvidos, visto as necessidades e fragilidades individuais à cada um.

Em se tratando do domínio totalidade e integração, ficou evidenciado que as crenças auxiliam a criar uma coerência (harmonia) entre o que se faz, pensa e sente $(62,2 \%)$ e que os profissionais estão satisfeitos por terem um equilíbrio entre a mente, o corpo e a alma (56,8\%). A espiritualidade, portanto, pode atuar como um fator de promoção da reflexão, estimulando a habilidade do ser humano de olhar para dentro de si e se manter resiliente frente às situações desafiadoras, promovendo assim força interior que favoreça o equilíbrio entre mente, corpo e alma (Hott, 2020). Deste modo, no cotidiano desses profissionais com pacientes infectados pela COVID-19, estarem apoiados a religiosidade/espiritualidade (RE), faz com que alguns benefícios sejam atingidos, tais como a melhor compreensão do seu papel frente à pandemia, a viabilidade de empregar suas experiências pessoais relacionadas a RE na melhoria do contato com os pacientes infectados, haja vista que o contato com estes é prejudicado pelo risco de transmissão (Scorsolini-comin et al., 2020).

Quanto ao domínio força espiritual, 50,5\% dos profissionais relatou sentir força espiritual interior, que a força espiritual os ajuda a viver melhor $(48,6)$ e a se sentir feliz na vida $(48,6 \%)$. Em um estudo realizado com 53 profissionais de saúde que atuam em uma Unidade de Terapia Intensiva, em uma cidade do interior da Bahia, os profissionais afirmaram que a sua religiosidade/espiritualidade exerce influência importante no entendimento de cada um quanto ao processo saúde doença, bem como na condução de sua relação com o paciente, portanto, a espiritualidade dos profissionais de saúde assume um importante papel, não somente sobre o cuidado prestado, como também proporcionando bem-estar, melhorando o relacionamento e a qualidade de vida de cada indivíduo (Longuiniere; Yarid; Silva, 2018).

No que diz respeito aos domínios paz interior e esperança e otimismo, respectivamente, 65,8\% dos profissionais relataram sentir um senso de harmonia na sua vida, conseguir sentir paz quando necessitam dela (64\%) e serem capazes de permanecer otimista em épocas de incerteza (64\%). Neste sentido, a espiritualidade, a fé e a religião suscitam sentimentos positivos, como o bem estar e o conforto, além de amenizar as situações de estresse (Oliveira et. al. 2018). Da mesma forma, diante de todas as dificuldades e complexidades impostas aos profissionais de saúde na realização do cuidado frente à COVID19, a prática da espiritualidade traz benéficos para saúde emocional, qualidade de vida e no envolvimento destes profissionais em suas atividades. Para tanto, em situações de crise, entender o sentido da vida dispondo-se da espiritualidade, pode tornar mais fácil o alcance da resiliência, ressignificação e esperança (Gomes et. al., 2020) Os achados nesse domínio corroboram com um estudo que revelou que saúde e espiritualidade não devem ser dissociados (Sant'ana; Silva; Vasconcelos, et al., 2020).

A pandemia causada pelo SARS-COV-2 trouxe grandes impactos nas diversas esferas e para toda a população global, sobretudo, no grupo de trabalhadores da área da saúde que atuam na linha de frente contra essa doença. Neste sentido, no tocante ao domínio fé, foi afirmado pela maior parte dos profissionais $(55,9 \%)$ que a fé os ajuda a gozar (aproveitar) a vida, destacando-se também o fato de a fé lhes dá força $(44,1 \%)$ e conforto $(42,3 \%)$ no dia-a-dia. Corroborando com os entrevistados da presente pesquisa, a fé é apresentada como um dos fatores que percorrem a espiritualidade, influenciando positivamente a saúde e resultando em esperança (Gerone, 2016). Do mesmo modo, a fé e a espiritualidade têm fundamental importância em ressignificar a vida, sendo geradoras de força e superação, tendo em vista as situações impossíveis de serem modificadas, mas passiveis de serem reestruturadas (Moura et. al.,2020).

Após serem calculadas, procedeu-se as correlações entre a espiritualidade e as variáveis idade, transtornos mentais comuns, qualidade de vida e medo da COVID.

Na Tabela 3 encontra-se as estatísticas dos instrumentos WHOQOL-SRBP, SF-12, QSG e Medo da COVID. 
Tabela 3 - Estatísticas dos instrumentos WHOQOL-SRBP, SF-12, QSG e Medo da COVID, entre profissionais de saúde do município de Vitória da Conquista, nos anos de 2020/2021.

\begin{tabular}{|c|c|c|c|c|}
\hline \multirow[b]{2}{*}{ Estatísticas } & \multicolumn{4}{|c|}{ Instrumentos } \\
\hline & WHOQOL-SRBP & SF-12 & QSG & Medo da COVID \\
\hline Valor mínimo & 48 & 11 & 9 & 8 \\
\hline Valor máximo & 127 & 25 & 36 & 39 \\
\hline Erro padrão & $1,5 \%$ & $0,33 \%$ & $0,9 \%$ & $0,82 \%$ \\
\hline Média & 102,04 & 19,94 & 22,53 & 23,11 \\
\hline Intervalo de Confiança & {$[99,07 ; 105,01]$} & {$[19,27 ; 20,60]$} & {$[20,72 ; 24,35]$} & {$[21,47 ; 24,76]$} \\
\hline Desvio Padrão & 15,81 & 3,07 & 7,21 & 6,84 \\
\hline
\end{tabular}

Fonte: Dados da pesquisa (2021).

Os escores apresentados pela espiritualidade variaram entre valor mínimo de 48 e máximo de 127, numa média de 102,04 e com um desvio padrão de 15,81. Com relação à idade, foram evidenciados valor mínimo de 21 e máximo de 57, com uma média de 36,69 e um desvio padrão de 7,15. Em se tratando do QSG, verificou-se variação dos escores entre 9 e 36, média de 22,53 e desvio padrão de 7,21. O SF 12 apresentou valores entre 11 e 25, média de 19,94 e desvio padrão de 3,07. O medo da COVID apresentou escores entre 8 e 39, média de 23,11 e um desvio padrão de 6,84.

Em virtude do tamanho da amostra foi utilizado o teste de normalidade Kolmogorov-Smirnov, sendo evidenciado que as variáveis espiritualidade $(\mathrm{p}=0,01)$, transtornos mentais comuns $(\mathrm{p}=0,00)$ e qualidade de vida ( $\mathrm{p}=0,004)$ não seguem distribuição normal, enquanto a variável medo da $\operatorname{COVID}(\mathrm{p}=0,2)$ apresenta-se como uma variável aleatória normal padrão.

$\mathrm{Na}$ Tabela 4 estão retratadas as correlações não-paramétricas que foram realizadas entre as variáveis, por meio do coeficiente de correlação de postos de Spearman.

Tabela 4 - Correlações de postos de Spearman entre espiritualidade, idade, transtornos mentais comuns e medo da COVID, entre profissionais de saúde do município de Vitória da Conquista, nos anos de 2020/2021.

\begin{tabular}{lcc}
\hline \multicolumn{1}{c}{ Variável } & Coeficiente de correlação & Significância \\
\hline Idade & 0,015 & $\mathrm{P}=0,874$ \\
Transtornos Mentais Comuns & 0,532 & $\mathrm{P}=0,000$ \\
Qualidade de vida & 0,211 & $\mathrm{P}=0,053$ \\
Medo da COVID & 0,142 & $\mathrm{P}=0,243$ \\
\hline
\end{tabular}

Fonte: Dados da pesquisa (2021).

Encontrou-se associação (21\%) entre a qualidade de vida e a espiritualidade ( $\mathrm{p}=0,053$ ), sendo evidenciado que a qualidade de vida aumenta com o maior índice de espiritualidade, resultado que corrobora com um estudo de revisão sistemática à respeito da espiritualidade/religiosidade em saúde no contexto brasileiro, onde foram avaliados 35 trabalhos e descrito o uso de 16 instrumentos para da dimensão espiritual da qualidade de vida, sendo o WHOQOL-SRPB um dos mais 
utilizados dentre os estudos (Forti et al., 2020). Os escores manifestados pelo módulo SRBP do WHOQOL apontaram correlações significativas com a qualidade de vida, sendo enfatizando a necessidade de se pesquisar e compreender a dimensão espiritual do ser humano (Forti et al., 2020). Da mesma forma, um estudo realizado entre 241 pessoas, fazendo uma comparação entre pessoas doentes e saudáveis, verificou a associação entre religiosidade e qualidade de vida, por meio da aplicação do WHOQOL-SRPB, demonstrando a influência da religiosidade sobre todos os domínios da qualidade de vida (psicológico, de relacionamentos sociais, ambientais e qualidade de vida em geral), havendo maior significância entre os escores apresentados pelo os doentes (Rocha; Fleck, 2011). Portanto, a dimensão espiritual do ser humano apresenta-se como uma aliada na busca pelo bem estar e no enfrentamento do processo saúde-doença.

Verificou-se uma correlação positiva (53\%) entre a espiritualidade e o desenvolvimento de transtornos mentais comuns $(\mathrm{p}=0,000)$, assim, o desenvolvimento de transtornos mentais comuns diminui à medida que aumenta a espiritualidade. Neste contexto, há que se considerar que dentre os estudos realizados sobre religiosidade/espiritualidade, cerca de $80 \%$ abrangem a saúde mental (Koenig, 2012). A inserção das práticas de espiritualidade/ religiosidade impactam positivamente na sua saúde mental de cada indivíduo, fazendo-se necessário que elas sejam reconhecidas pelos profissionais de saúde como um recurso promotor de saúde mental (Oliveira; Junges, 2012). Tais práticas auxiliam na redução do estresse e das emoções negativas, ao mesmo tempo em que proporcionam o apoio social e afetam de maneira positiva os comportamentos relacionados à saúde (Oliveira; Junges, 2012). Assim, verifica-se que a importância dos aspectos relacionados à espiritualidade no âmbito da saúde mental tem sido cada vez mais discutida, a fim de se abordar a saúde de cada pessoa de forma global, envolvendo tanto as questões físicas quanto mentais o que se aplica à saúde ocupacional do profissional da saúde, diante de toda a complexidade vivenciada frente à COVID-19. Não foi evidenciado correlação significativa entre a espiritualidade e a idade ( $p=0,87$, coeficiente de correlação 0,015 ), bem como entre a espiritualidade e o medo da COVID ( $p=0,243$, coeficiente de correlação 0,142$)$.

\section{Conclusão}

Este estudo desvelou a necessidade de compreender o papel significativo que a espiritualidade tem no suporte para o enfrentamento às situações de incertezas e de grandes mudanças decorrentes da pandemia atual. Portanto, as práticas de espiritualidade atuam como promotoras da qualidade de vida dos profissionais que estão na linha de frente dos serviços de saúde, os quais estão diariamente expostos ao contágio, tendo que lidar com o medo das possíveis consequências que possam surgir. Evidenciou-se que a espiritualidade está relacionada ao menor índice de transtornos mentais comuns e à maior qualidade de vida.

Os resultados encontrados estão de acordo com a literatura, porém, considera-se como limitação deste estudo a quantidade reduzida de profissionais respondentes, o que pode ser justificado pelo fato de estes estarem na linha de frente do cenário pandêmico, apresentando-se num importante grau de exaustão física e emocional, além do grande número de pesquisas que estão sendo realizadas neste período, sendo os mesmos profissionais incluídos e convidados a participar. Além disso, há de se considerar a probabilidade de respostas automáticas, sendo uma consequência ao cenário em questão.

Trata-se de uma pesquisa inovadora acerca da temática, sendo possível que os resultados auxiliem na reflexão e formulação de estratégias que apoiem a promoção da saúde e do bem-estar dos profissionais em questão, os quais têm a espiritualidade como suporte no exercício diário da profissão. Faz-se necessário a realização de novos estudos sobre a temática, de uma forma mais abrangente, nos diferentes contextos nacionais e internacionais, de forma que seja possível discutir, numa 
perspectiva global, estratégias de enfrentamento utilizadas pelos profissionais de saúde frente à pandemia da COVID-19, apresentando mecanismos de promoção de qualidade de vida aos profissionais em questão.

\section{Referências}

Alves, J. S., Junges, J. R. \& López, L. C. (2010). A dimensão religiosa dos usuários na prática do atendimento à saúde: percepção dos profissionais da saúde. O Mundo da Saúde, São Paulo; 34(4):430-436.

Barros, M. N., Aguiar, M. M., Carvalho, F., Macedo, A. \& Pereira, A. T. (2021). Escala de Medo da COVID-19 - Validação e adaptação para o Período Perinatal. J Hum Growth Dev.; 31(1):09-17.

Bentzen, J. (2020). In Crisis, We Pray: Religiosity and the COVID-19 Pandemic. CEPR: Economia Pública (Tema).57p.

Borges, L. O. \& Argolo, J. C. T. (2002). Adaptação e validação de uma escala de bem-estar psicológico para uso em estudos ocupacionais. Avaliação Psicológica, 1, pp. 17-27.

Camelier, A. A. (2004). Avaliação da qualidade de vida relacionada à saúde em pacientes com DPOC: estudo de base populacional com o SF-12 na cidade de São Paulo-SP [tese]. São Paulo: Universidade Federal do Estado de São Paulo.

Casella, I. B. (2020). Fisiopatologia da trombose associada à infecção pelo SARS-CoV-2. J Vasc Bras;19: e 20200128. https://doi.org/10.1590/16775449.200128

Catré, M. N. C., Ferreira, J. A., Pessoa, T., Pereira, M., Canavarro, M. C. \& Catré, A. (2014). O domínio SRPB (Spirituality, Religiousness and Personal Beliefs) do WHOQOL: O estudo com grupos focais para validação da versão em Português europeu do WHOQOL-SRPB. Análise Psicológica, 4 (XXXII): 401-417. Doi: 10.14417/ap.872.

Cespedes, M. S. \& Souza, J. C. R. P. (2020). SARS-CoV-2: uma revisão para o clínico. Rev Assoc Med Bras; 66(2):116-123

Chan, J., Yuan, S., Kok, K., et. al. (2020). A familial cluster of pneumonia associated with the 2019 novel coronavirus indicating person-to-person transmission: a study of a family cluster. Lancet: Feb 15, Vol 395: 514-23.

Chu, H., Chan, J., Yuen, T., Shuai, H., Yuan, S., Wang, Y., Hu, B., Yip, C.C., Tsang, J.O., Huang, X., et. al. (2020). Comparative tropism, replication kinetics, and cell damage profiling of SARS-CoV-2 and SARS-CoV with implications for clinical manifestations, transmissibility, and laboratory studies of COVID19: an observational study. Lancet Microbe; 1: e14-23, abr.

Ferrer, R. (2020). Pandemia por COVID-19: el mayor reto de la historia del intensivismo. Med Intensiva; 44:323-324.

Fiho, J. M. J., Assunção, A. Á., Algranti, E., Garcia, E. G., Saito, C. A. \& Maeno, M. (2020). A saúde do trabalhador e o enfrentamento da COVID-19. Rev Bras Saude Ocup; 45 : e14.

Fleck, M. P. \& Skevington, S. (2007). Explicando o significado do WHOQOL-SRPB. / Rev. Psiq. Clín. 34, supl 1; $146-149$.

Forti, S., Serbena, C.A. \& Scaduto, A.A. (2020). Mensuração da espiritualidade/religiosidade em saúde no Brasil: uma revisão sistemática. Ciência \& Saúde Coletiva, 25(4):1463-1474.

Gerone, L.G.T. (2016). A religiosidade/espiritualidade na prática do cuidado entre profissionais da saúde. Interações - cultura e comunidade, Belo Horizonte, Brasil, V.11 N.20, P. 129-151, Jul./Dez.

Gomes, A. M. T., Yarid, S. D., Silva, A. E., Nogueira, V. P. F. \& França, L. C. M. (2020). Apontamentos de pesquisa: a pandemia COVID-19- teologia, ciência e arte em conversas Vol. V/ organização de Everton Nery Carneiro, Sandra Célia Coelho Gomes da Silva, Luis Távora Furtado Ribeiro - Curitiba: Brazil Publishing. [recurso eletrônico].

Hott, M. C. M. (2020). COVID-19: a espiritualidade harmonizando saúde mental e física. J. Health Biol Sci.; 8(1):1-3.

Kendall, E. J., Bynoe M. L. \& Tyrrell D. A. (1962). Isolamentos de vírus de resfriados comuns que ocorrem em uma escola residencial. Br Med J.; 2 (5297): 82-86. Doi: $10.1136 /$ bmj.2.5297.82

Koenig, H. G. (2012). Religion, spirituality, and health: the research and clinical implications. ISRN Psychiatry.

Li, G. \& De Clercq, E. (2020). Therapeutic options for the 2019 novel coronavirus (2019-nCoV). Nat Rev Drug Discov. Mar;19(3):149-150. Doi: 10.1038/d41573-020-00016-0. PMID: 32127666.

Longuiniere, A. C. F., Yarid, S. D. \& Silva, E. C. S. Influência da religiosidade/espiritualidade do profissional de saúde no cuidado ao paciente crítico. Rev Cuid.9(1):1961-72.

Lu, R., Zhao, X., Li, J., Niu, P., et.al. (2020). Genomic characterisation and epidemiology of 2019 novel coronavirus: implications for virusoriginsand receptor binding. Lancet. Feb 22;395(10224):565-574;

Zhang, W. Manual de Prevenção e Controle da Covid-19 segundo o Doutor Wenhong Zhang. $1^{\text {a }}$ ed. São Paulo-SP: Polobooks. 
Melo, C. F., Sampaio, I. S., Souza, D. L. A. \& Pinto, N.S. (2015). Correlação entre religiosidade, espiritualidade e qualidade de vida: uma revisão de literatura. Estudos e Pesquisas em Psicologia. Rio de Janeiro v. 15 n. 2 p. 447-464.

Minayo, M. C. S. \& Sanches, O. Quantitativo-Qualitativo: Oposição ou Complementaridade? Cad. Saúde Públ. Rio de Janeiro, 9 (3): $239-262$, jul/set, 1993.

Moura, H. C. G. B, Menezes, T. M. O., Freitas, R. A., Moreira, F. A., Pires, I. B, Nunes, A. M. P. B., et al. (2020). Faith and spirituality in the meaning of life of the elderly with chronic kidney disease. Rev Bras Enferm;73(Suppl 3): e20190323. Doi: http://dx.doi.org/10.1590/0034-7167-2019-0323

Narayanasamy, A. (2002). Spiritual coping mechanisms in chronically ill patients. BRITISH JOURNAL OF NURSING, VOL $11, \mathrm{~N}^{\circ} 22$.

Oliveira, M. R. \& Junges J. R. (2012). Saúde mental e espiritualidade/religiosidade: a visão de psicólogos. Estudos de Psicologia, 17(3), setembro-dezembro, 469-476.

Oliveira, D. S., Furtado, L. N. S., Azevedo, F. B., Ramos, S. B. \& Bittar, C. M. L. (2018). Influência da espiritualidade, religiosidade e crenças pessoais na qualidade de vida de pacientes em quimioterapia. Temas em Saúde, Volume 18, Número 2 ISSN 2447-2131 João Pessoa.

Panzini, R. G., Maganha, C., Rocha, N. S., Bandeira, D. R. \& Fleck, M. P. (2011). Validação brasileira do Instrumento de Qualidade de Vida/espiritualidade, religião e crenças pessoais. Rev Saude Publica;45(1):153-65.

Rocha, N. S. \& Fleck, M. P. A. (2011). Avaliação de qualidade de vida e importância dada a espiritualidade/religiosidade/ crenças pessoais (SRPB) em adultos com e sem problemas crônicos de saúde. Rev Psiq Clín;38(1):19-23

Rouquayrol, M. Z. (1994). Epidemiologia \& Saúde. Rio de Janeiro: Medsi Editora Médica e Científica Ltda.

Sant'ana, G., Silva, C.D. \& Vasconcelos, M. B. A. (2020). Espiritualidade e a pandemia da COVID-19: um estudo bibliográfico. Comunicação em Ciências da Saúde, v. 31, n. 03, p. 71-77.

Scorsolini-Comin F., Rossato L., Cunha V. F., et al. (2020). A Religiosidade/Espiritualidade como Recurso no Enfrentamento da Covid-19. Revista de Enfermagem do Centro Oeste Mineiro;10: e3723; http://doi.org/10.19175/recom.v10i0.3723

Silveira, M. F., Almeida, J. C., Freire, R. S., Haikal, D. S. \& Martins, A. E. B. L. (2013). Propriedades psicométricas do instrumento de avaliação da qualidade de vida: 12-item health survey (SF-12). Ciência \& Saúde Coletiva, 18(7):1923-1931.

Strabelli, T. M. V \& Uip, D. (2020). COVID-19 e o Coração. Arq Bras Cardiol; 114(4):598-600.

Su, S., Wong, G. \& Shi, W., et al. (2016). Epidemiology, genetic recombination, and pathogenesis of coronaviruses.Trends Microbiol; 24: 490-502;

Tavares, C. Q. (2020). Dimensões do cuidado na perspectiva da espiritualidade durante a pandemia pelo novo coronavírus (COVID-19). J Health NPEPS; $5(1): 1-4$.

Underwood, L. G. (1999). A Working Model of Health. Journal of Religion, Disability \& Health, 3: 3, 51-71, https://doi.org/doi: 10.1300 / J095v03n03_05

Wu, F., Zhao, S., Yu, B., et. al. (2020). A new coronavirus associated with human respiratory disease in China. March. Nature 579, 265-269 (2020). https://doi.org/10.1038/s41586-020-2008-3

Yee, J., Unger, L., Zadravecz, F., Cariello, P., Seibert, A., Johnson, M.A. \& Matthew J.F. (2020). Novel coronavirus 2019 (COVID-19): Emergence and implications for emergency care. JACEP Open; 1:63-69.

Zhang, H., Penninger. J.M., Li, Y., Zhong, N. \& Slutsky, A.S. (2020). Angiotensin-converting enzyme 2 (ACE2) as a SARS- CoV-2 receptor: molecular mechanisms and potential therapeutic target. Intensive Care Med.; 46:586- 590 